\title{
3D anthropometric assessment and perception of male body morphology in relation to physical strength
}

\author{
Tobias L. Kordsmeyer ( ) I Julia Stern ｜ Lars Penke
}

Department of Psychology \& Leibniz ScienceCampus Primate Cognition, University of Goettingen, Goettingen, Germany

\section{Correspondence \\ Tobias L. Kordsmeyer, Department of Psychology \& Leibniz ScienceCampus Primate Cognition, University of Goettingen, Gosslerstr. 14, 37073 Goettingen, Germany. \\ Email: tob.kor@gmail.com}

\section{Funding information}

Deutsche Forschungsgemeinschaft, Grant/

Award Number: Project number 254142454/GRK 2070; Leibniz-

Gemeinschaft, Grant/Award Number: Leibniz ScienceCampus Primate Cognition

\begin{abstract}
Objectives: The assessment of men's physical strength is an important part of human social perception, for which observers rely on different kinds of cues. However, besides previous studies being limited in considerable ways, as yet there is no comprehensive investigation of a range of somatometric measures in relation to both objectively measured and observer-perceived physical strength using valid stimuli.

Methods: We examined observer-perceptions of physical strength from 3D body scans of $n=165$ men, the usage and validity of somatometric measures as cues to strength, differences between strength ratings from stimuli presented on computer monitors vs in real-life size using a projector, and between male and female observers.

Results: A medium-sized correlation between measured and perceived strength was found, partly mediated by target men's chest-to-hip ratio, body density, ankle girth, height, upper arm, and forearm girth. No significant differences between men's and women's strength perceptions or the method of stimuli presentation (computer monitor vs projector) emerged.

Conclusions: Our findings suggest that men's physical strength can be assessed with moderate accuracy from 3D body models and that some somatometric measures represent valid cues, which were used by observers, positively predicting both measured and perceived physical strength.
\end{abstract}

\section{1 | INTRODUCTION}

The assessment of men's physical strength is an important part of human social perception, whether as an indicator of social dominance (Maner, DeWall, \& Gailliot, 2008), fighting ability (Sell et al., 2009) or generally resource acquisition potential in the evolutionary past (Sell, Lukaszewski, \& Townsley, 2017). Sell et al. (2009) suggested that specific cognitive mechanisms for the assessment of physical formidability (ie, the ability to inflict physical costs on others, Durkee, Goetz, \& Lukaszewski, 2018) have evolved under selection pressures of a high prevalence of aggressive social encounters in men (see also Durkee et al., 2018 for recent evidence that physical strength is rapidly and automatically perceived by observers, $\mathrm{n}=64$ target men, $\mathrm{n}=187$ male and female raters). These mechanisms may be rooted in more general, cross-species adaptive benefits of assessing an opponent's fighting ability before entering a contest. In his seminal work on the animal kingdom, Parker (1974) argued that individuals adjust their fighting strategy (eg, to fight or withdraw) based on their own resource holding potential (RHP, defined as the ability to win a contest, independent of motivation) as well as opponents' RHP (eg, Arnott \& Elwood, 2009; Stulp, Kordsmeyer, Buunk, \& Verhulst, 2012). Male intrasexual competition has played a central role throughout human evolution in the distribution of resources, such as mates, territory and food, and eventually in the evolution of secondary sexual traits and agonistic behaviors, such as dominance (defined as the use of coercive behaviors to induce fear and enforce one's will, Puts, 2016; Puts, Bailey, \& Reno, 2015). Thus, possessing 
physical strength arguably has always been more important for males than for females (Sherlock, Tegg, Sulikowski, \& Dixson, 2016). In men, higher body strength correlates positively with important life outcomes, such as mating success $(n=4774$, Lassek \& Gaulin, 2009) and occupational success, particularly in pre-industrial societies (Eagly \& Wood, 1999). The higher prevalence of intrasexual competition in men is one potential explanation for men's higher physical strength (Archer, 2009), which in turn poses a potential threat not only to other men, but also to women (eg, sexual abuse, threat to their offspring, Smuts, 1992). Hence, the assessment of men's, compared to women's, physical formidability purportedly has been more relevant throughout human evolutionary history for both men and women (Rudman \& Goodwin, 2004). In line with this, Sell et al. (2009) showed that both men and women more accurately judged physical strength of men, compared to women (overall $n=245$ target participants). In this study, we aimed at extending these findings by investigating the perception of men's physical strength by both male and female observers employing naturalistic stimuli, how accurately these perceptions reflect objectively measured strength, and how different observable body cues mediate associations between perceived and actual strength.

When assessing physical strength, observers can rely on a range of visual and auditory cues (eg, body height and weight, $\mathrm{n}=118$ target men and women, $\mathrm{n}=60$ raters, Holzleitner \& Perrett, 2016; Sell et al., 2009; vocal characteristics, $\mathrm{n}=221$ men and women, Han et al., 2018; $\mathrm{n}=111$ target men, $\mathrm{n}=86$ male raters, Puts, Gaulin, \& Verdolini, 2006; facial dominance and attractiveness, $\mathrm{n}=32$ target men, $\mathrm{n}=79$ female raters, Fink, Neave, \& Seydel, 2007; gait, $n=20$ target men, $n=101$ raters, Fink et al., 2016). In Sell and colleagues' study (2009), observers could reliably predict target men's physical strength from facial and body photographs, using cues such as upper body muscularity besides height, weight, and age. Holzleitner and Perrett (2016) used three-dimensional (3D) face scans to investigate specific facial cues that correlate with strength assessments. A large amount of variance in ratings of strength from male faces could be explained by cues related to body size, muscle mass, and fat mass. A further body measure which might account for strength perceptions of male bodies is waist-to-chest ratio (WCR). In a study by Coy, Green, and Price (2014), female raters ascribed higher physical dominance, physical fitness, and protection ability to male bodies with lower WCR (ratings based on avatars created from $n=153$ D body scans, $n=151$ female raters). Hence, a larger upper, relative to the lower, torso was positively related to perceptions of dominance and physical strength (see also Beagan \& Saunders, 2005; Flynn, Park, Morin, \& Stana, 2015).

Previous studies on perceptions of men's physical strength and related traits were limited in that they only focused on a small selection of somatometric measures (eg, WCR in Coy et al., 2014; muscularity, height, and weight in Sell et al., 2009; and facial/auditory characteristics such as voice pitch in Puts et al., 2006). Hence, so far there is a gap in the literature of a comprehensive investigation of which body characteristics explain strength perceptions, and which body characteristics are related to objectively measured physical strength. Sell et al. (2009) examined the relationship between measured and observer-perceived physical strength and found positive correlations. However, in their studies only frontal body photographs were used, which are limited in ecological validity, since in real-life assessments of physical strength should not only be based on a frontal perspective (Sell et al., 2017 used 2D body photographs including both frontal and side views for strength and attractiveness judgments). In our study, we aimed to replicate and extend these findings by examining somatometric measures (eg, several girth measures) and combined indices (eg, WCR) as correlates of perceived and measured physical strength. Our study comes with some unique strength. First, we used a relatively large number of realistic morphological stimuli from 3D body scans. This method has been proposed to become the new standard in anthropometry (Jaeschke, Steinbrecher, \& Pischon, 2015; Kuehnapfel, Ahnert, Loeffler, Broda, \& Scholz, 2016; Löffler-Wirth et al., 2016), and has already been successfully applied in previous studies of strength (eg, Holzleitner \& Perrett, 2016; Price, Dunn, Hopkins, \& Kang, 2012) and attractiveness perception (Mautz, Wong, Peters, \& Jennions, 2013; Price, Pound, Dunn, Hopkins, \& Kang, 2013; Smith, Cornelissen, \& Tovée, 2007). Jaeschke et al. (2015) showed that automatic measures by means of $3 \mathrm{D}$ body scanning have high validity and reliability in comparison to traditional manual measurement $(n=60)$. Moreover, Kuehnapfel et al. (2016) found that the intrarater and interrater reliability of automatic measurements based on 3D body scans are comparable to those of manual measurements $(\mathrm{n}=108)$. Furthermore, full-body 3D scans allow for a large number of somatometric measures to be considered as cues, potentially mediating the association between observer perceived and measured physical strength. Second, two presentation methods of the stimuli were compared (traditional computer monitor vs life-size projection) to examine if the scale of stimuli presentation has an influence on observerperceptions. Mautz et al. (2013) already successfully employed a life-size presentation of body stimuli in their study on the influence of penis size on male attractiveness.

We focused on the following main research questions: firstly, can men's physical strength be perceived by males and females from body scans in a reliable and valid way? Secondly, which somatometric measures are used as cues and to what extent are they valid for predicting measured strength? Thirdly, is strength perceived differently when body stimuli are presented on comparably small computer monitors vs in real-life size using a projector? Finally, are there sex differences in the accuracy of strength perceptions (Sell et al., 2009 found a 
slightly higher accuracy for male than for female raters; Holzleitner \& Perrett, 2016 and Sell et al., 2017 found no significant effect of rater sex, 2 samples, overall $n=192$ target men, $\mathrm{n}=219$ raters) or in cue usage? We predicted physical strength to be positively related to strength ratings, for both male and female raters. Our analyses were rather exploratory concerning which somatometric measures (in the following referred to as body cues) exactly predict strength perceptions and measured strength. Based on previous studies, we hypothesized strength ratings to be positively related to body height and weight (Holzleitner \& Perrett, 2016; Sell et al., 2009), and WCR (Coy et al., 2014).

\section{2 | METHODS}

\section{1 | Participants}

Participants were $\mathrm{n}=165$ male heterosexual young adults (age: $M=24.3, S D=3.2$, range 18-34 years), mostly recruited from the graduate and undergraduate student population at the University of Göttingen (Germany) (88.5\% students, 98.8\% European ethnicity). Eighty indicated single status, 85 were in a relationship (11 open, 66 committed, 4 engaged, 4 married, none divorced or widowed). On the 7-point Kinsey scale of sexual identity $(1=$ exclusively heterosexual to $7=$ exclusively homosexual; Kinsey, Pomeroy, \& Martin, 1948), the mean was 1.19 ( $S D=0.46)$. All participants signed an informed consent form and the study received approval from the local ethical committee (number 111).

\section{2 | Body scan measurements}

Participants were scanned three times using the Vitus ${ }^{\text {smart }}$ XXL bodyscanner running AnthroScan software (both Human Solutions $\mathrm{GmbH}$, Kaiserslautern, Germany), while wearing standardized tight underwear. Participants were instructed to stand in a standardized position, upright with legs hip-widely apart, arms stretched out and held slightly away from the body, making a fist with thumbs showing forward, the head positioned in accordance with the Frankfort horizontal, and to breathe normally during the scanning process (ca. 10 seconds).

AnthroScan's automatic measures (all according to ISO 20685:2005, German Institute for Standardization, 2006) include the following parameters purportedly relevant to body muscularity: mid-neck girth (AnthroScan \#1510), waist girth (6510), bust-chest girth (4510), hip girth (7520*), upper arm girth (left: 8520 \& right: 8521), forearm girth (8540 \& 8541), thigh girth (9510 \& 9521), ankle girth (9550 \& 9551), calf girth (9540 \& 9541), and inside-leg-ankle length (9010 \&

*In AnthroScan, this measure was named as "buttock girth", but we took it as the hip girth measure, because we believed this measure came closest to the waistband and hence what we wanted to measure as hip girth.
9011). In addition to automatic measurements, biacromial shoulder width was measured manually (on screen) as the direct distance between the left and right acromion processes. Reliabilities for the three body scans were high for all measures (intraclass correlations, two-way random, single measures $>$.90; for a complete list see Table S1 in the supplementary), and comparable to previous research (Jaeschke et al., 2015). We calculated waist-to-hip ratio (WHR), shoulder-to-hip ratio (SHR), waist-to-chest ratio (WCR), chest-to-hip ratio (CHR), and leg length-to-height ratio (LHR). An aggregate indicator of upper body size was calculated by averaging $z$-standardized shoulder width, bust-chest girth, and upper arm girth (means of left and right arms, see Price et al., 2012). For body cues, averages of the three body scans for each participant were used, and for bilateral traits, the means of left and right measures. The volumes (in liters) of 15 body parts (head, upper torso, lower torso, and both hands, forearms, upper arms, thighs, calves, and feet) were measured from one body scan of each participant (the first of the three; for some cases the measurement of body volume did not work and the second or third scan was used). Total body volume was measured from scans, and body density was approximated by dividing body mass by body volume (Goldman \& Buskirk, 1961).

\section{3 | Physical strength measurements}

Physical strength was operationalized as upper body and handgrip strength. Handgrip strength was shown to be highly correlated with overall body strength (Wind, Takken, Helders, \& Engelbert, 2010) and to play a role in interpersonal perception (Fink, Neave, \& Seydel, 2007). Both handgrip and upper body strength were measured using a hand dynamometer (Saehan SH5001). Each measurement was taken three times, starting with handgrip strength with the handle adjusted to the second position, for which participants were asked to use their dominant hand $(88.2 \%$ used their right, the remaining $11.8 \%$ their left hand). For upper body measures, the handle was inverted and moved to the outermost position (see Sell et al., 2009); participants held the dynamometer in front of their chest with both hands and pressed both handles toward the middle as strongly as possible. For both handgrip and upper body strength measures, participants were asked to start putting pressure on the dynamometer slowly to prevent a biased measure by pushing too rapidly, after which they were supposed to use full force. Between attempts, participants were allowed to take a short rest to account for muscle fatigue. Of the three measures, the maximum values obtained were used as the strength indicators for handgrip and upper body strength separately. A composite measure of physical strength was formed by averaging the two maximum values after $z$-standardization. Reliabilities 
were acceptable to good (intraclass correlations: .81 and .64 for handgrip and upper body strength, respectively).

Body height (in centimeters) was measured twice using a stadiometer, while participants stood upright barefooted, and the two values were averaged. Weight (in kilograms) was measured three times as part of each body scanning process with the integrated scale SECA 635 (SECA, Hamburg, Germany); the three values were averaged. Body-mass index (BMI) was calculated from average weight and height measures $\left(\mathrm{kg} / \mathrm{cm}^{2}\right)$.

\section{4 | Rating study}

\subsection{1 | Raters}

A total of $\mathrm{n}=121$ raters (61 males; age: $M=25.1, S D=6.1$, range $18-53$ years) were recruited at the University of Göttingen. In addition to sex and age, their profession (or study subject), relationship status (57 single, 6 in an open relationship, 49 in a committed relationship, 3 engaged, 4 married, 2 others), and sexual orientation (using the 7-point Kinsey scale) were assessed.

\subsection{2 | Stimuli creation}

Of the originally $n=165$ target men, 13 had to be excluded due to parts of long scalp hair visible in the neck and shoulder region (which would bias relevant somatometric measures), leaving a final sample of $n=152$. From each of the target men, one body scan was chosen by visual inspection (ie, the scan coming closest to the standardized posture).

Body scans did not contain information on skin texture or color, but only morphological cues presented in standardized gray color, because they were based on laser technology. To create an even body surface removing small holes in the surface from the scanning procedure, body scans were converted using the function "Surface Reconstruction of Standard Scan" of AnthroScan software and exported as (Wavefront) *.OBJ files. Body scans were truncated above the neck using the software Blender (version 2.75, www.blender.org), leaving an even plane just below the larynx. This was done in order to focus raters' attention on bodily features and to preserve anonymity of target male participants. In case a participant had a pronounced trapezius muscle, the cutoff line was moved upwards slightly in the neck region in order to leave this muscle as a whole, while leaving it underneath the larynx up front (thus creating a cutoff line bent down towards the front). Finally, animated videos of a body scan turning around its vertical axis were created ("beauty turns", duration: 8 seconds each; $960 \times 540$ pixels). The 152 beauty turns were divided into two sets of 76 videos matched for BMI. Thus, we obtained two sets, which were similar both in mean and variation of body composition, as indicated by BMI.

\subsection{3 | Presentation of stimuli}

One group of raters viewed the stimuli on a $24^{\prime \prime}$-computer monitor. A second group rated the stimuli from projections on a white wall (cf. Mautz et al., 2013). This was done to test whether real-life size presentations are comparable to those presented on a small screen. In both settings, the software Alfred (Treffenstaedt \& Wiemann, 2018), based on the programming language Python (version 2.7; www.python. org), was used for stimuli presentation. For each set, a preview of all 76 beauty turns ( 1 second each) was presented to familiarize raters with the stimulus material and range of bodies. Stimuli were presented randomly and participants indicated their ratings on paper. Participants were instructed to watch the beauty turns until they had completed one full turn of $360^{\circ}$ before providing their rating. Videos were presented in an infinite loop, so that participants could decide when to move to the next stimulus. In the computer monitor condition, ratings were conducted in rooms with a maximum of four computers. The projector ratings were situated in a larger room with maximum of six raters present at one time.

\subsection{4 | Strength ratings}

Perceived strength was assessed by raters using the item "How physically strong is this man?" on an 11-point Likertscale ranging from -5 (very weak) to +5 (very strong). Between 14 and 16 male and female raters judged each body in each of the two conditions (for details, see Table 1). Ratings were averaged by target men, separately for male and female raters for the computer monitor and projector conditions. Interrater reliabilities were high across conditions and rater sex (intraclass correlations, average two-way random agreement $>.90$ for both conditions).

\section{5 | Statistical analyses}

All variables were $z$-standardized to zero mean and unit variance. To assess differences in male and female ratings and between the two modes of presentation (computer monitor vs projector), correlations of strength ratings with measured strength (aggregate of handgrip and upper body strength) and somatometric measures were compared using Fisher $z$-transformation (Fisher, 1915). Pearson correlations between body cues and both measured and perceived strength were calculated. Multiple linear regression models were performed, with the dependent variables measured and observerperceived strength (in two separate models) and body cues as the independent variables, to assess which of the body cues uniquely predicted measured and perceived strength. Selection of independent variables for the regression models was done in an exploratory way, initially excluding variables from the pool of potential predictors, which had an effect neither 
TA B LE 1 Detailed distribution of and information about the raters

\begin{tabular}{lll} 
& Male raters & Female raters \\
\hline Computer monitor condition & $(1): 14 ;(2): 16$ & $(1): 16 ;(2): 14$ \\
\hline Projector condition & $(1): 15 ;(2): 16$ & $(1): 15 ;(2): 15$ \\
\hline Age & $M=25.8, S D=6.6$ & $M=24.4, S D=5.4$ \\
Age range & $18-53$ & $19-48$ \\
$\%$ single & 45.9 & 48.3 \\
\hline
\end{tabular}

Note: $(1) /(2)=$ stimuli set $1 / 2$. on perceived nor measured strength. Among the remaining body cues, we aimed at including the variables for which the regression models showed high amounts of explained variance $\left(R^{2}\right)$ and simultaneously low variance inflation factors (VIFs).

Brunswikian lens model analyses were conducted, to analyze the utilization and validity of somatometric measures as cues for the link between measured and perceived strength (Brunswik, 1956; Nestler \& Back, 2013). Measured strength served as the criterion (underlying trait) and was related to body cues for assessing to what extent these cues predict the former (cue validity). The link between perceived strength and body cues provides insights into which somatometric measures are used (and to what extent) by observers when assessing target men's physical strength (cue utilization). Moreover, the association between perceived and measured strength was assessed (accuracy, Nestler \& Back, 2013). To formally assess mediating effects by body cues on the association between perceived and measured strength, mediator analyses were conducted using the lavaan package in R (R Core Team, 2015; Rosseel, 2012).

\section{3 | RESULTS}

\section{1 | Descriptive statistics and bivariate correlations}

Descriptive statistics for all variables, including all somatometric measures (including left and right sides), the three strength measures (handgrip, upper body and the aggregate measure), and all four strength ratings (male and female raters, in both the computer monitor and projector conditions) plus two aggregated ratings (males and females averaged across both conditions) are provided in the supplementary (Table S2). Bivariate Pearson correlations between all variables are reported in Table S3.

\section{2 | Comparing associations across strength measures, rating condition and rater sex}

Associations of body cues with handgrip strength and upper body strength revealed no significant differences (Fisher $z$ transformation, all unsigned $z \mathrm{~s}<1.21, p \mathrm{~s}>.22$ ). Thus, for a more robust measure of physical strength, we decided to use aggregate strength as the average of handgrip and upper body strength for our analyses. When comparing the ratings in the computer monitor and projector condition, no significant differences in correlations of somatometric measures with perceived strength emerged, neither for male (all unsigned $z \mathrm{~s}<1.46, p \mathrm{~s}>.14)$ nor for female raters $(z \mathrm{~s}<0.56$, $P \mathrm{~s}>.57)$. Thus, in the following analyses, only computer monitor ratings were used. When comparing male and female ratings in the computer monitor conditions, no significant differences in correlations of perceived strength with somatometric measures were detected (all unsigned $z \mathrm{~s}<1.18$, $p s>.23$ ). The correlation between strength ratings of males and females in the computer monitor condition was high $(r=.94, p<.001)$. Hence, in the following an average measure of both ratings was used.

\section{3 | Linear regression models predicting measured and perceived strength}

The final models (for the two dependent variables measured and perceived strength separately), which showed a large amount of explained variance $\left(R^{2}\right)$ and at the same time low variance inflation factors (VIFs) for the independent variables, comprised the predictors body height, chest-tohip ratio (CHR), upper arm girth, waist-to-hip ratio (WHR) and body density. Results revealed that shorter men with a lower WHR, a higher CHR, higher body density and larger upper arm girth were perceived as stronger (Table 2). Men who were taller, had a higher body density, as well as larger upper arm girth, were measured to be stronger (no significant effect of WHR and CHR; Table 3). The predictor variables explained 65\% of the variance (adjusted $R^{2}$ ) in perceived strength, and $21 \%$ in measured strength, and all VIFs were low $(<1.40)$. Thus, upper arm girth as well as body density positively predicted both perceived and measured strength, and CHR positively predicted perceived strength. WHR was a negative predictor for perceived but not measured strength, whereas height was associated positively with measured strength and negatively with perceived strength. 
T A B L E 2 Linear regression models predicting perceived strength

\begin{tabular}{|lrrrrl} 
& \multicolumn{1}{c}{$\boldsymbol{\beta}$} & $\boldsymbol{S} \boldsymbol{E}$ & \multicolumn{1}{l}{$\boldsymbol{t}$} & $\boldsymbol{p}$ & $\boldsymbol{V I F}$ \\
\hline Height & -.22 & 0.53 & -4.17 & $<.001$ & 1.20 \\
\hline Chest-to-hip ratio & .50 & 0.57 & 8.83 & $<.001$ & 1.39 \\
\hline Upper arm girth & .47 & 0.55 & 8.55 & $<.001$ & 1.31 \\
\hline Waist-to-hip ratio & -.51 & 0.56 & -9.15 & $<.001$ & 1.33 \\
\hline Body density & .23 & 0.52 & 4.40 & $<.001$ & 1.15 \\
\hline
\end{tabular}

Note: Perceived strength: males and females aggregated, computer monitor condition; model fit: $F_{5,146}=56.61, p<.001, R^{2}=.66$, adjusted $R^{2}=.65$. Abbreviation: VIF, variance inflation factor.

T A B LE 3 Linear regression models predicting measured strength

\begin{tabular}{lrlccc} 
& $\boldsymbol{\beta}$ & $\boldsymbol{S} \boldsymbol{E}$ & \multicolumn{1}{c}{$\boldsymbol{t}$} & $\boldsymbol{p}$ & $\boldsymbol{V} \boldsymbol{V} \boldsymbol{F}$ \\
Height & .19 & 0.79 & 2.44 & .02 & 1.20 \\
Chest-to-hip ratio & .16 & 0.85 & 1.92 & .057 & 1.39 \\
\hline Upper arm girth & .30 & 0.83 & 3.63 & $<.001$ & 1.31 \\
\hline Waist-to-hip ratio & -.07 & 0.84 & -0.82 & .41 & 1.33 \\
\hline Body density & .20 & 0.78 & 2.58 & .01 & 1.15 \\
\hline
\end{tabular}

Note: Measured strength $=$ handgrip and upper body strength aggregated; model fit: $F_{5,146}=9.05, p<.001, R^{2}=.24$, adjusted $R^{2}=.21$.

Abbreviation: VIF, variance inflation factor.

\section{4 | Lens model analyses}

Brunswikian lens model analyses revealed that the following body cues were positively related to both rated and measured strength: bust-chest girth, upper arm girth, forearm girth, BMI, CHR, upper body size, and body density (Figures 1 and 2). A number of variables were associated positively with measured, but not perceived strength: weight, mid-neck girth, waist girth, hip girth, thigh girth, ankle girth, calf girth, and shoulder width. Leg length and WCR were used by raters as cues (negatively), but were unrelated to measured strength.

\section{5 | Mediation analyses}

Mediation analyses revealed significant indirect effects on the association between perceived and measured strength by upper arm girth, forearm girth, CHR, body density (all positive), as well as height and ankle girth (both negative; Table 4). This means the correlation between perceived and measured strength would decrease considerably when keeping constant men's upper arm girth, forearm girth, CHR and body density, but would increase when controlling for height and ankle girth.

Based on significant positive effects of body density on both perceived and measured strength in the lens model analyses, and since body density is unlikely to be perceived directly by observers, we wanted to further investigate which of the other body cues partly explained associations of body density with perceived strength. Mediation analyses showed positive indirect effects of CHR, upper arm and forearm girth on perceived strength (indirect effects $=.15 / .15 / .11$, $S E \mathrm{~s}=.05 / .05 / .05, z \mathrm{~s}=2.80 / 2.85 / 1.98, p \mathrm{~s}=<.01 /<.01 / .047$; Table S4). Hence, body density was positively associated with CHR, upper arm and forearm girth, which led to higher strength ratings by observers (for lens model analyses, see Figure 3).

\section{4 | DISCUSSION}

This study examined the relationship between men's observerperceived and measured physical strength, and potential mediating effects of morphological body cues, including various somatometric measures, body volume and density. Results showed a medium-sized correlation between measured and perceived strength, suggesting that men's physical strength can be assessed with moderate accuracy from 3D body models. No significant differences were found between males' and females' ratings and when displaying the bodies on a computer monitor vs in real-life size using a projector. Brunswikian lens model analyses showed that out of all 22 somatometric measures, the following turned out to be positively related to both perceived and measured strength: bust-chest girth, upper arm and forearm girth, BMI, chest-to-hip ratio (CHR), upper body size, and body density. Largely converging with these associations, mediation analyses showed that the correlation between perceived and measured physical strength was partly explained by CHR, body density, upper arm and forearm girth (positively), as well as ankle girth and height (negatively). Hence, these somatometric measures represent valid cues, which positively predict men's measured physical strength, and at the same time were used by observers judging strength (except for height and ankle girth, which were related positively to measured strength only, and in the case of height negatively to perceived strength).

Thigh, ankle and calf girth positively predicted measured strength but were not used by our observers judging strength. Presumably, observers focused their attention on the upper body, for which we detected medium-sized correlations with strength ratings. Some of these upper body measures, in turn, positively predicted measured strength (bustchest girth, upper arm and forearm girth, CHR, upper body size). Leg length, however, inversely predicted perceived strength (unrelated to measured strength), which relates to the surprising negative association between body height and perceived strength. These two findings likely present an oddity of this sample, despite its rather large size $(n=152)$, especially since previous studies found positive associations of strength ratings with body height (Holzleitner \& Perrett, 2016; Sell et al., 2009; Undurraga, Zebrowitz, Eisenberg, Reyes-Garcia, \& Bolivia Study Team, 2012). Negative correlations of body 


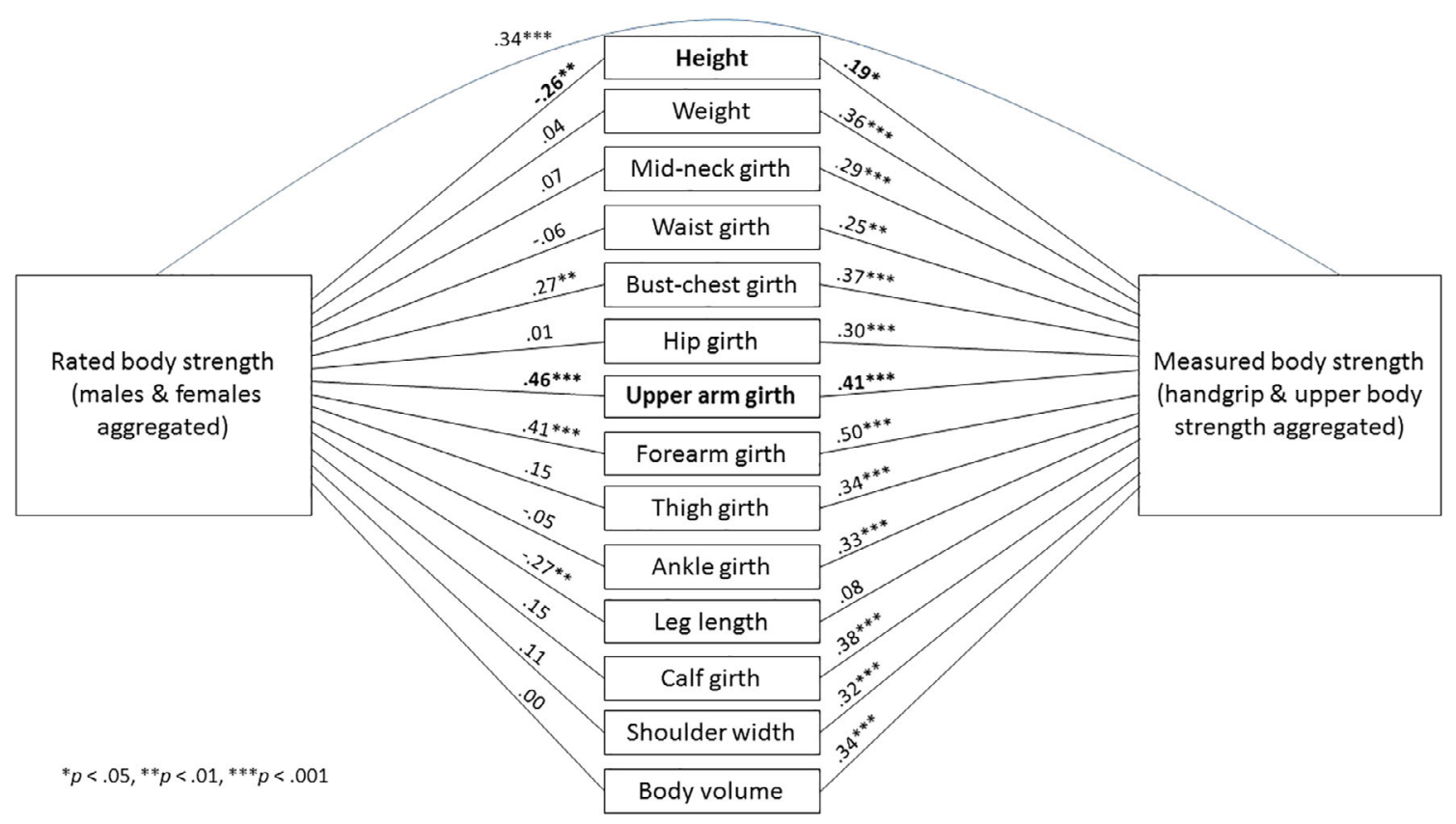

F I G U RE 1 Lens model depicting Pearson correlations between simple somatometric measures and perceived (left) and measured (right) strength. For bilateral traits, the means of left and right sides were used here. Variables included in the regression models are printed in bold

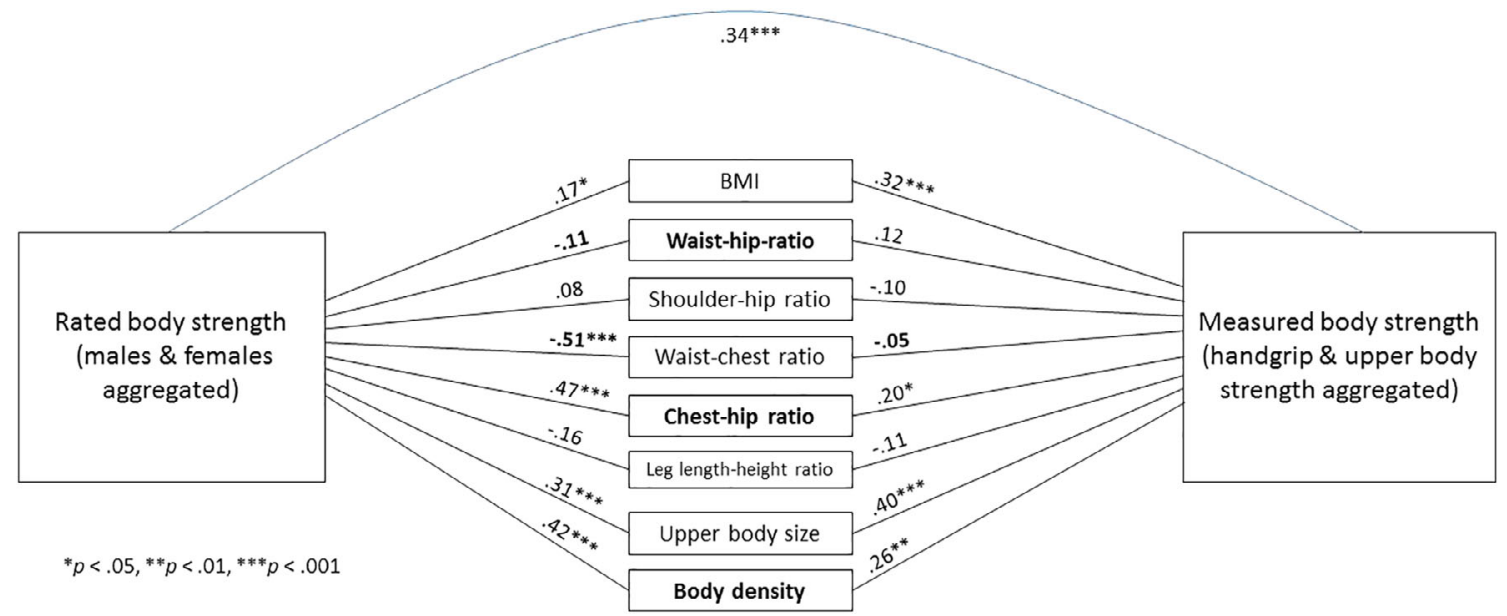

F I G URE 2 Lens model showing Pearson correlations between measured body indices and perceived (left) and measured (right) strength. Variables included in the regression models are printed in bold

height and leg length were also found when only considering strength perceptions based on real-life size projection, which should have yielded an increased salience of height differences among target men, relative to the computer monitor condition. As measured strength was positively related to body height (in line with Sell et al., 2009; see Holzleitner \& Perrett, 2016 for a null finding), it seems this sample of target men is characterized by a slight overrepresentation of short men who look stronger than they were measured to be, and tall men who looked weaker than they were. Thus, this study's findings regarding the link between height and perceived strength should be treated with care.
In the study by Coy et al. (2014), male bodies with lower WCR were perceived as higher in physical dominance, physical fitness, and protection ability by females, all concepts directly linked to physical strength. While we were able to show a similar link of WCR with perceived strength, WCR was not associated with measured strength. That is, our raters relied on WCR for their strength judgments, which did not appear to be a valid cue to measured strength. Furthermore, the shoulder-to-hip ratio (SHR), which is strongly inversely related to WCR, was not linked with perceived or measured physical strength, questioning earlier findings. For example, Gallup, White and Gallup Jr. (2007) found a positive association 
T A B L E 4 Mediation analyses for association between perceived and measured strength; body cues as mediators

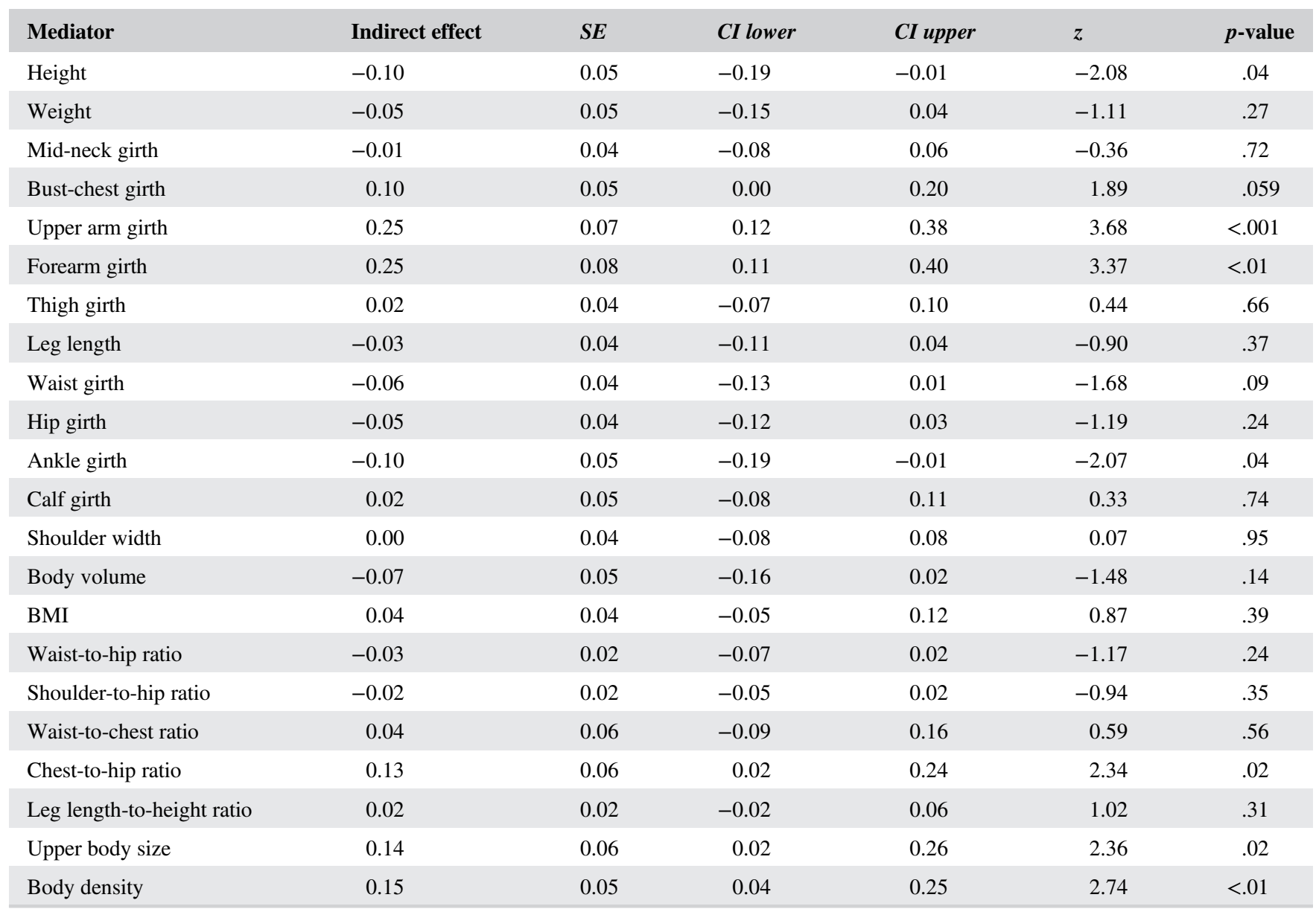

Abbreviation: CI, confidence interval. $* p<.05$.

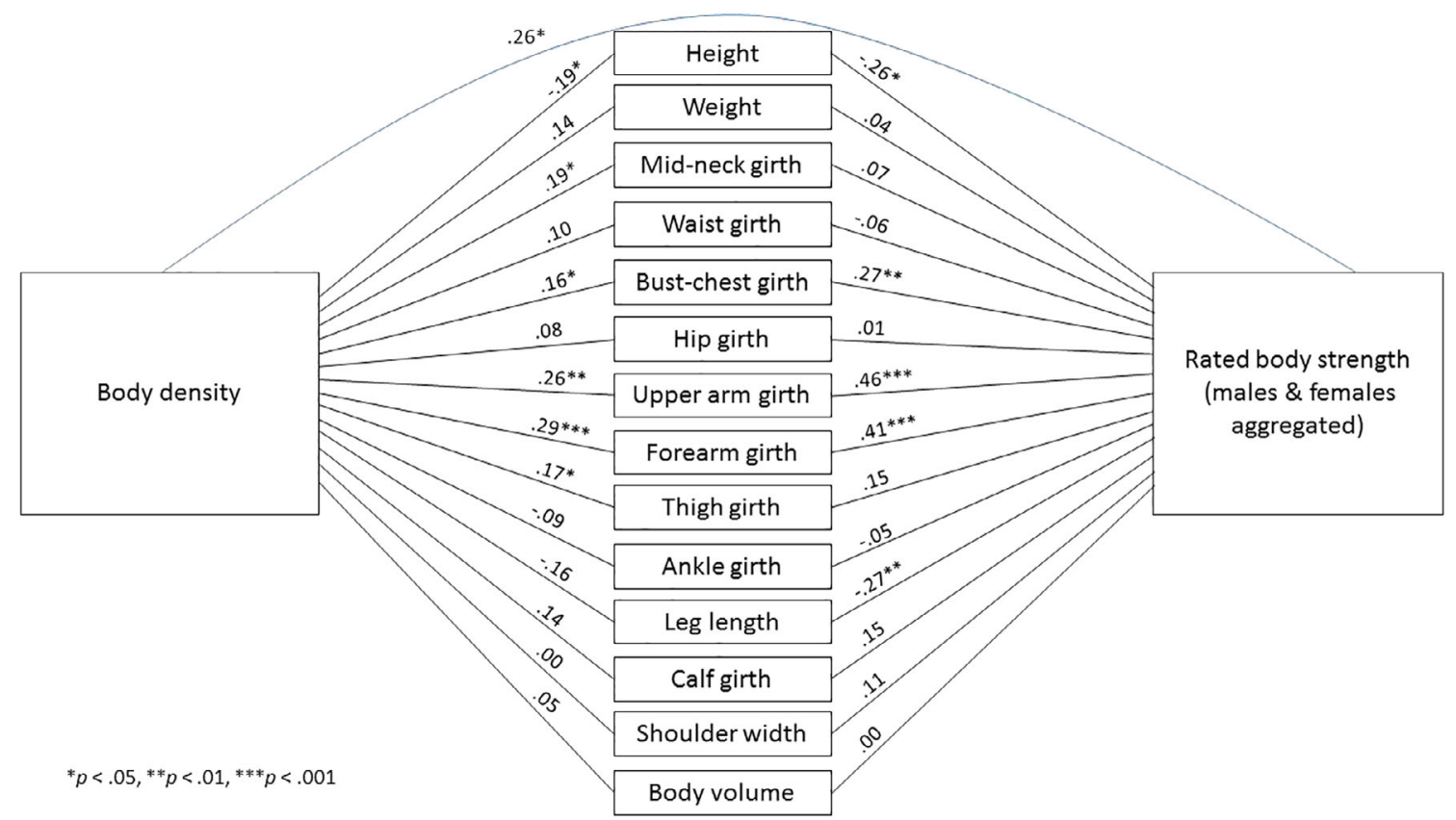

F I G U R E 3 Lens model depicting Pearson correlations between simple somatometric measures and body density (left) and perceived strength (right). For bilateral traits, the means of left and right sides were used here 
between SHR and handgrip strength in male college students $(\mathrm{n}=82)$. However, in their study the effect was significant only for left-hand, but not right-hand, measures, and shoulder circumference was measured, in contrast to biacromial shoulder width in our study. Still, our lack of findings for SHR and WCR may question the validity of men's upper bodies' "vshape" or "inverted triangle" shape (ie, narrow hip and waist and wide shoulders and chest) as cues to physical strength (cf. Maisey, Vale, Cornelissen, \& Tovée, 1999). A further variable which intuitively is well implicated in strength measures and perceptions is an individual's body weight. Holzleitner and Perrett (2016) distinguished between muscle and fat mass, and both were positively related to strength ratings, and the former to measured strength. In Sell and colleagues' study (2009), contradictive findings emerged regarding weight and strength perceptions. For ratings of full-person and body-only images, associations were either negative or non-significant, whereas, for face-only stimuli, correlations were positive or non-significant. In our study, weight was unrelated to perceived strength, but positively correlated with measured strength. Thus, we provide some support for an association between body weight and measured strength, but a potential link with strength perceptions requires further investigation. Relatedly, Holzleitner and Perrett (2016) found BMI to be positively related to both perceived and measured strength. We were able to replicate both effects; target men with higher BMI were both measured and perceived to be physically stronger, on average. BMI appears to be a valid cue to physical strength, which is used by observers, in contrast to height and weight. Moreover, Durkee et al. (2018) used eye-tracking data to analyze which body regions male and female observers look at when judging men's physical strength (from frontal full-body photos, with target men wearing wide shorts). They found that observers primarily viewed target men's faces and upper bodies, especially the chest regions, and hardly looked at the legs. This is in line with our findings of positive associations between observerperceived strength and chest girth, upper arm and forearm girth (but not thigh and calf girth), among others. Thus, it seems there is considerable evidence that observers especially focus on the upper body, more so than on the leg region, when assessing men's physical strength.

A measure which, to our knowledge, has not been examined in relation to measured or perceived strength is body density. In our study, men with a higher body density (ie, higher weight for a given volume) were both measured and perceived to be physically stronger. This effect remained significant after controlling for further somatometric measures, hence it likely represents a promising candidate for a valid cue in objective and subjective assessments of physical strength. Since body density is unlikely to be perceived directly by observers due to its relatively low variance, we further investigated which somatometric measures are related to body density and serve as cues to perceived strength. Mediation analyses revealed that men with a higher body density also had larger upper arm and forearm girths, as well as a higher CHR, which led to increased strength perceptions. This shows that men's body density may act as an indirectly observable cue to physical strength, and that perceivers may infer physical strength from it based on arm and body torso measures. Further research is required to determine the robustness of these effects. Also, the reliability of our method of assessing body density (especially of one of its main components, body volume) deserves further attention, such as by comparing it with more traditional ways of measurement (eg, Fuller, Jebb, Laskey, Coward, \& Elia, 1992; Jackson, Pollock, \& Ward, 1980).

Thus, regarding associations of somatometric measures with observer-perceived and measured physical strength, we provide support for some previous findings (eg, WCR, BMI), but contradicting evidence for others (eg, height, partly weight), and suggest a new measure, body density, which in our study showed associations with both perceived and measured physical strength. Moreover, even though all leg measures (except for leg length) were associated with measured strength, raters did not rely on these for their strength judgments, but rather on upper body measures, such as bust-chest girth, upper arm and forearm girth, and indices like BMI, WCR, CHR, upper body size, and body density. We also found more and somewhat stronger associations of somatometric measures with measured physical strength than with strength ratings, and vice versa for body indices; it seems our observers integrated information from at least two somatometric measures for their strength judgments, more so than relying on single measures.

The correlation between perceived and measured physical strength in this study was positive and statistically significant, but only medium-sized ( $r=.34$ ). In Sell and colleagues' studies $(2009,2017)$, observers showed a higher accuracy in judging men's strength $(r=.57-.66$ in Sell et al., 2009; $r=.45-.61$ in Sell et al., 2017). While our stimuli material can be seen as at least as valid as theirs (revolving 3D body scans vs 2D static images of bodies), the operationalization of physical strength differed somewhat. Sell and colleagues used either a combination of upper body strength with flexed bicep measurements and self-reported strength, aggregated performance on four weight-lifting machines, or chest compression and handgrip strength. Thus, it may be that the strength measures of Sell and colleagues better mapped aspects of physical strength that are perceptible to observers, than our combination of handgrip and upper body strength. If these differences turned out to be robust, our study on somatometric measures as cues to strength perceptions could be repeated, employing more multi-facetted strength measures.

This study also aimed at comparing the strength judgments of male and female raters. Previous studies reported either no sex differences (Holzleitner \& Perrett, 2016; Sell et al., 2017) 
or only a slightly higher accuracy for male than for female raters (Sell et al., 2009). In line with the former, we found no significant differences in correlations between perceived strength and somatometric measures, and a very high correlation between males' and females' strength ratings. Pending limited generalizability from this mostly student sample, our finding here may be in line with the notion that the assessment of men's physical formidability is similarly important for both men and women (for women, among others threat to their offspring or of sexual abuse, Smuts, 1992, but also for assessing mate value and resource acquisition potential; for men, related to the high prevalence of intra-sexual competition, for example, Archer, 2009). Another objective of this study was to compare two rating conditions (computer monitor vs real-life size using a projector). We detected no significant differences in correlations between single somatometric measures and perceived strength, undermining Mautz et al.' (2013) claim that presenting life-size stimuli rather than small versions might yield different estimates.

Our study employed observer-perceptions of physical strength based on naturalistic 3D body models created from 3D body scans, instead of 2D images (Sell et al., 2009) or 3D avatars of male bodies (Coy et al., 2014), rendering our ratings more externally valid (though it should be noted that our scans only contained morphological features, but no information on skin texture or color). The somatometric measures were also derived from these 3D body scans, a quick and efficient method of body measurement (Kuehnapfel et al., 2016), for which a high validity and reliability has been demonstrated (Jaeschke et al., 2015). Moreover, in our rating study we compared two modes of stimuli presentation, of which one was to present the body scan videos in real-life size (eg, Mautz et al., 2013) and another on regular computer monitors (eg, Sell et al., 2009).

One main limitation of this study is the lack of female body stimuli. We decided to focus on male targets only, because the assessment of physical formidability has been more relevant in men than women throughout human evolution (Rudman \& Goodwin, 2004). In a follow-up study, observer-perceptions of female body strength could be assessed, to compare the pattern of associations between strength perceptions, measured strength and somatometric measures with those found for male targets. The finding of Sell et al. (2009) of a higher accuracy of strength judgments for male than female targets could be replicated. Furthermore, the age range of our target men was somewhat restricted (18-34 years). A follow-up study could employ a wider age range, which would render results more generalizable. A particularly interesting sample may be adolescents, since this age group typically shows the highest propensity to aggressiveness (Dahlberg, 1998). A further interesting addition would be to examine the influence of observer characteristics. The influence of observers' age (George, Swami, Cornelissen, \& Tovée,
2008), personality (Swami et al., 2012) and even current ecological conditions (Swami \& Tovee, 2007) have been investigated in previous studies on attractiveness perceptions, but not strength perceptions. Observers' sensitivity to target men's physical strength might as well depend on their own physical strength (see Durkee et al., 2018 for an initial finding of a positive association between raters' self-perceived formidability and their ratings of targets' strength, though in one out of two conditions only) and experience with sports or fitness training, for instance.

\section{5 | CONCLUSIONS}

In this study on the link between observer-perceived and measured physical strength in men, a range of somatometric measures were found as valid cues to strength, that is, were positively related to both perceived and measured strength. These were bust-chest girth, upper arm and forearm girth, BMI, chest-to-hip ratio, upper body size, body density and height (albeit the latter surprisingly showed a negative link with perceived strength). Certain body cues were only related to observer-perceived, but not measured strength (WCR and, negatively, leg length), and vice versa for others (weight, mid-neck, waist, hip, thigh, ankle, and calf girth, biacromial shoulder width, and body volume). Overall, it appeared that observers based their strength judgments more on men's upper body measures than those from the leg regions. Furthermore, we did not detect any sex difference between male and female raters in associations between perceived strength and body cues, nor an influence of stimuli presentation mode (in real-life size using a projector vs on computer monitors). These findings provide further insights into an important facet of human interpersonal perception, namely how men's physical strength is perceived by male and female observers, which body cues are used for these judgments, and how perceived strength is related to objectively measured strength.

\section{ACKNOWLEDGMENTS}

This research was funded by the Deutsche Forschungsgemeinschaft (DFG, German Research Foundation, Project number 254142454 / GRK 2070) and by the Leibniz-Gemeinschaft through funding for the Leibniz ScienceCampus Primate Cognition. The authors thank Maximilian Eberhard for support in stimuli creation, and Dr. Bernhard Fink for providing comments on an earlier version of the manuscript.

\section{AUTHOR CONTRIBUTIONS}

TLK, JS and LP designed the study. TLK and JS collected the data. TLK analyzed the data and drafted the manuscript. LP and JS revised the manuscript. 


\section{ORCID}

Tobias L. Kordsmeyer (10 https://orcid.org/0000-0003-27429176

\section{REFERENCES}

Archer, J. (2009). Does sexual selection explain human sex differences in aggression? Behavioral and Brain Sciences, 32, 249-266. https://doi.org/10.1017/S0140525X09990951

Arnott, G., \& Elwood, R. W. (2009). Assessment of fighting ability in animal contests. Animal Behaviour, 77, 991-1004. https://doi.org/ 10.1016/j.anbehav.2009.02.010

Beagan, B., \& Saunders, S. (2005). Occupations of masculinity: Producing gender through what men do and don't do. Journal of Occupational Science, 12, 161-169. https://doi.org/10.1080/14427591. 2005.9686559

Brunswik, E. (1956). Perception and the representative design of psychological experiments (2nd ed.). Berkeley, CA: University of California Press.

Coy, A. E., Green, J. D., \& Price, M. E. (2014). Why is low waist-tochest ratio attractive in males? The mediating roles of perceived dominance, fitness, and protection ability. Body Image, 11, 282-289.

Dahlberg, L. L. (1998). Youth violence in the United States: Major trends, risk factors, and prevention approaches. American Journal of Preventive Medicine, 14, 259-272. https://doi.org/10.1016/ S0749-3797(98)00009-9

Durkee, P. K., Goetz, A. T., \& Lukaszewski, A. W. (2018). Formidability assessment mechanisms: Examining their speed and automaticity. Evolution and Human Behavior, 39, 170-178. https://doi. org/10.1016/j.evolhumbehav.2017.12.006

Eagly, A. H., \& Wood, W. (1999). The origins of sex differences in human behavior: Evolved dispositions versus social roles. American Psychologist, 54, 408-423. https://doi.org/10.1037/0003-066X. 54.6.408

Fink, B., André, S., Mines, J. S., Weege, B., Shackelford, T. K., \& Butovskaya, M. L. (2016). Sex difference in attractiveness perceptions of strong and weak male walkers. American Journal of Human Biology, 28, 913-917. https://doi.org/10.1002/ajhb.22891

Fink, B., Neave, N., \& Seydel, H. (2007). Male facial appearance signals physical strength to women. American Journal of Human Biology, 19, 82-87. https://doi.org/10.1002/ajhb.20583

Fisher, R. A. (1915). Frequency distribution of the values of the correlation coefficient in samples from an indefinitely large population. Biometrika, 10, 507-521. https://doi.org/10.2307/2331838

Flynn, M. A., Park, S. Y., Morin, D. T., \& Stana, A. (2015). Anything but real: Body idealization and objectification of MTV docusoap characters. Sex Roles, 72, 173-182. https://doi.org/10.1007/s11199015-0464-2

Fuller, N. J., Jebb, S. A., Laskey, M. A., Coward, W. A., \& Elia, M. (1992). Four-component model for the assessment of body composition in humans: Comparison with alternative methods, and evaluation of the density and hydration of fat-free mass. Clinical Science, 82, 687-693. https://doi.org/10.1042/cs0820687

Gallup, A. C., White, D. D., \& Gallup, G. G. (2007). Handgrip strength predicts sexual behavior, body morphology, and aggression in male college students. Evolution and Human Behavior, 28, 423-429. https://doi.org/10.1016/j.evolhumbehav.2007.07.001
George, H. R., Swami, V., Cornelissen, P. L., \& Tovée, M. J. (2008). Preferences for body mass index and waist-to-hip ratio do not vary with observer age. Journal of Evolutionary Psychology, 6, 207-218. https://doi.org/10.1556/JEP.6.2008.3.4

German Institute for Standardization (2006). ISO 20685:2005: 3-D scanning methodologies for internationally compatible anthropometric databases. German version EN ISO 20685:2005. Retrieved from https://www.iso.org/standard/35514.html.

Goldman, R. F., \& Buskirk, E. R. (1961). Body volume measurement by underwater weighing: Description of a method. In J. Brozek \& A. Henschel (Eds.), Techniques for measuring body composition (pp. 78-106). Washington, DC: National Research Council, National Academy of Sciences.

Han, C., Wang, H., Fasolt, V., Hahn, A. C., Holzleitner, I. J., Lao, J., ... Jones, B. C. (2018). No clear evidence for correlations between handgrip strength and sexually dimorphic acoustic properties of voices. American Journal of Human Biology, 30, e23178. https://doi. org/10.1002/ajhb.23178

Holzleitner, I. J., \& Perrett, D. I. (2016). Perception of strength from 3D faces is linked to facial cues of physique. Evolution and Human Behavior, 37, 217-229. https://doi.org/10.1016/j.evolhumbehav.2015. 11.004

Jackson, A. S., Pollock, M. L., \& Ward, A. N. N. (1980). Generalized equations for predicting body density of women. Medicine and Science in Sports and Exercise, 12, 175-181.

Jaeschke, L., Steinbrecher, A., \& Pischon, T. (2015). Measurement of waist and hip circumference with a body surface scanner: Feasibility, validity, reliability, and correlations with markers of the metabolic syndrome. PLoS One, 10, e0119430. https://doi.org/10.1371/ journal.pone.0119430

Kinsey, A. C., Pomeroy, W. B., \& Martin, C. E. (1948). Sexual behavior in the human male. Philadelphia: W. B. Saunders.

Kuehnapfel, A., Ahnert, P., Loeffler, M., Broda, A., \& Scholz, M. (2016). Reliability of 3D laser-based anthropometry and comparison with classical anthropometry. Scientific Reports, 6, 1-11. https://doi.org/10.1038/srep26672

Lassek, W. D., \& Gaulin, S. J. (2009). Costs and benefits of fat-free muscle mass in men: Relationship to mating success, dietary requirements, and native immunity. Evolution and Human Behavior, 30, 322-328. https://doi.org/10.1016/j.evolhumbehav.2009. 04.002

Löffler-Wirth, H., Willscher, E., Ahnert, P., Wirkner, K., Engel, C., Loeffler, M., \& Binder, H. (2016). Novel anthropometry based on 3D-bodyscans applied to a large population based cohort. PLoS One, 11, e0159887. https://doi.org/10.1371/journal.pone. 0159887

Maisey, D. S., Vale, E. L., Cornelissen, P. L., \& Tovée, M. J. (1999). Characteristics of male attractiveness for women. The Lancet, 353, 1500. https://doi.org/10.1016/S0140-6736(99)00438-9

Maner, J. K., DeWall, C. N., \& Gailliot, M. T. (2008). Selective attention to signs of success: Social dominance and early stage interpersonal perception. Personality and Social Psychology Bulletin, 34, 488-501. https://doi.org/10.1177/0146167207311910

Mautz, B. S., Wong, B. B., Peters, R. A., \& Jennions, M. D. (2013). Penis size interacts with body shape and height to influence male attractiveness. Proceedings of the National Academy of Sciences, 110, 6925-6930. https://doi.org/10.1073/pnas.1219361110

Nestler, S., \& Back, M. D. (2013). Applications and extensions of the lens model to understand interpersonal judgements at zero 
acquaintance. Current Directions in Psychological Science, 22, 374-379. https://doi.org/10.1177/0963721413486148

Parker, G. A. (1974). Assessment strategy and the evolution of fighting behaviour. Journal of Theoretical Biology, 47, 223-243. https:// doi.org/10.1016/0022-5193(74)90111-8

Price, M. E., Dunn, J., Hopkins, S., \& Kang, J. (2012). Anthropometric correlates of human anger. Evolution and Human Behavior, 33, 174-181. https://doi.org/10.1016/j.evolhumbehav.2011. 08.004

Price, M. E., Pound, N., Dunn, J., Hopkins, S., \& Kang, J. (2013). Body shape preferences: Associations with rater body shape and sociosexuality. PLoS One, 8, e52532. https://doi.org/10.1371/ journal.pone.0052532

Puts, D. (2016). Human sexual selection. Current Opinion in Psychology, 7, 28-32. https://doi.org/10.1016/j.copsyc.2015.07.011

Puts, D. A., Bailey, D. H., \& Reno, P. L. (2015). Contest competition in men. In D. M. Buss (Ed.), The Handbook of Evolutionary Psychology (Vol. 1: Foundations; pp. 385-402). Hoboken, New Jersey: John Wiley \& Sons. https://doi.org/10.1002/9781119125563. evpsych113

Puts, D. A., Gaulin, S. J., \& Verdolini, K. (2006). Dominance and the evolution of sexual dimorphism in human voice pitch. Evolution and Human Behavior, 27, 283-296. https://doi.org/10.1016/j. evolhumbehav.2005.11.003

R Core Team (2015). R: A language and environment for statistical computing. R Foundation for Statistical Computing, Vienna, Austria. Retrieved from https://www.R-project.org

Rosseel, Y. (2012). Lavaan: An R package for structural equation Modeling. Journal of Statistical Software, 48, 1-36. https://doi.org/ 10.18637/jss.v048.i02

Rudman, L. A., \& Goodwin, S. A. (2004). Gender differences in automatic in-group bias: Why do women like women more than men like men? Journal of Personality and Social Psychology, 87, 494-509. https://doi.org/10.1037/0022-3514.87.4.494

Sell, A., Cosmides, L., Tooby, J., Sznycer, D., von Rueden, C., \& Gurven, M. (2009). Human adaptations for the visual assessment of strength and fighting ability from the body and face. Proceedings of the Royal Society of London B, 276, 575-584. https://doi.org/10. 1098/rspb.2008.1177

Sell, A., Lukaszewski, A. W., \& Townsley, M. (2017). Cues of upper body strength account for most of the variance in men's bodily attractiveness. Proceedings of the Royal Society of London B, 284, 20171819. https://doi.org/10.1098/rspb.2017. 1819

Sherlock, J. M., Tegg, B., Sulikowski, D., \& Dixson, B. J. (2016). Facial masculinity and beardedness determine men's explicit, but not their implicit responses to male dominance. Adaptive Human
Behavior and Physiology, 3, 1-16. https://doi.org/10.1007/s40750016-0047-7

Smith, K. L., Cornelissen, P. L., \& Tovée, M. J. (2007). Color 3D bodies and judgements of human female attractiveness. Evolution and Human Behavior, 28, 48-54. https://doi.org/10.1016/j.evolhumbehav. 2006.05.007

Smuts, B. (1992). Male aggression against women. Human Nature, 3, 1-44. https://doi.org/10.1007/BF02692265

Stulp, G., Kordsmeyer, T., Buunk, A. P., \& Verhulst, S. (2012). Increased aggression during human group contests when competitive ability is more similar. Biology Letters, 8, 921-923. https://doi. org/10.1098/rsbl.2012.0591

Swami, V., Stieger, S., Pietschnig, J., Voracek, M., Furnham, A., \& Tovée, M. J. (2012). The influence of facial piercings and observer personality on perceptions of physical attractiveness and intelligence. European Psychologist, 17, 213-221. https://doi.org/10. 1027/1016-9040/a000080

Swami, V., \& Tovee, M. J. (2007). Differences in attractiveness preferences between observers in low-and high-resource environments in Thailand. Journal of Evolutionary Psychology, 5, 149-160. https:// doi.org/10.1556/JEP.2007.1005

Treffenstaedt, C., \& Wiemann, P. (2018). Alfred - A library for rapid experiment development [Survey software] (Version 0.2b5). https:// doi.org/10.5281/zenodo.1437220

Undurraga, E. A., Zebrowitz, L., Eisenberg, D. T. A., ReyesGarcia, V., \& Bolivia Study Team, T. A. P. S. (2012). The perceived benefits of height: Strength, dominance, social concern, and knowledge among Bolivian native Amazonians. PLoS One, 7, e35391. https://doi.org/10.1371/journal.pone.0035391

Wind, A. E., Takken, T., Helders, P. J., \& Engelbert, R. H. (2010). Is grip strength a predictor for total muscle strength in healthy children, adolescents, and young adults? European Journal of Pediatrics, 169, 281-287. https://doi.org/10.1007/s00431-009-1010-4

\section{SUPPORTING INFORMATION}

Additional supporting information may be found online in the Supporting Information section at the end of this article.

How to cite this article: Kordsmeyer TL, Stern J, Penke L. 3D anthropometric assessment and perception of male body morphology in relation to physical strength. Am J Hum Biol. 2019;e23276. https://doi.org/10.1002/ajhb.23276 